\section{Kontaktallergien und Dialyse}

\author{
Obwohl bei der Blutwäsche viele Substanzen zum Einsatz kommen, \\ deren sensibilisierende Potenz bekannt ist, gab es über die Häufigkeit \\ von Kontaktallergien bei Dialysepatienten bisher kaum Daten. Diesen \\ Mangel haben französische Autoren jetzt ausgeglichen.
}

Ä rzte des Hôpital Sainte Marguerite in Marseille untersuchten 75 Dialysepatienten ohne bekannte Hautkrankheiten zunächst dermatologisch: Sie fanden in $8 \%$ der Fälle ein auf den Shuntbereich lokalisiertes Ekzem. 16\% der 75 Patienten klagten außerdem über Juckreiz an dieser Prädilektionsstelle.

Es folgte eine Epikutantestung der 75 Patienten. Dabei wurde auf eine von der europäischen Kontaktdermatitisgruppe vorgeschlagene Testreihe zurückgegriffen, die durch weitere Allergene ergänzt wurde, die von der französischen Forschungsgruppe „Gerda“ empfohlen werden. Hinzugenommen wurden außerdem dialysespezifische Testallergene. Insgesamt umfasste das Testprogramm 53 Allergene. Bei einem positiven Epikutantestergebnis für ein Allergen wurde dann von einer klinischen Aktualität ausgegangen, wenn Allergenkontakt an einer ekzematisierten Hautstelle bestanden hatte und nach Allergenkarenz Besserung eingetreten war.

Bei 25\% der getesteten Dialysepatienten fanden sich ein oder mehrere positive Hauttests. 17\% der Patienten hatten dabei auf Allergene aus dem Dialyseumfeld reagiert, mit $11 \%$ am häufigsten auf ein Lidocain-Prilocain-Gemisch, welästhesie vor der Gefäßpunktionen aufgetragen wird. In der Sensibilierungshäufigkeit folgten Desinfektions- und Konservierungsmittel. Auffällig war, dass in keinem Fall eine Sensibilisierung gegen Chlorhexidin dokumentiert werden konnte und dass die Sensibilisierungsrate gegenüber dem am häufigsten zur Hautdesinfektion verwendeten Jodpovidon mit $4 \%$ sehr niedrig war.

\section{Fazit}

Kontaktsensibilisierungen sind bei französischen Dialysepatienten mit einer Prävalenz von $25 \%$ häufig. Ähnliches dürfte auch für deutsche Verhältnisse gelten. Lokalanästhetika, Desinfektionsund Konservierungsmittel sind dabei die wichtigsten Sensibilisierungsquellen. Pruritus und Kontaktekzem lokalisieren sich im Shuntbereich. Eine Ekzemstreuung wurde nicht beobachtet.

\section{Dr. Dieter Bruchhausen, Wuppertal}

\section{Literatur \\ Gaudy-Marqueste C, Jouhet C, Castelain M, Brunet P, Berland Y, Grob JJ, Richard MA. Contact allergies in haemodialysis patients: a prospective study of 75 patients. Allergy 2009; 64: 222-8}

ches als Creme regelmäßig zur Hautan-
Allergologie im Kloster 2010

„Porta patet, cor magis“, zu deutsch „Die Tür steht offen, mehr noch das Herz!" - getreu diesem Wahlspruch der Zisterzienser wird im Kloster Eberbach seit dem Jahre 1116 eine weltoffene und gastfreundliche Tradition gelebt. Seit einigen Jahren dürfen wir Allergologen jeweils im Wonnemonat Mai bei der Veranstaltung „Allergologie im Kloster" in diese Tradition eintauchen. Dieses Jahr findet das Treffen am 1. Mai statt. Wichtige Themen 2010 sind:

— Warum juckt die Haut?

- Allergieprävention im Kindesalter: Was hilft wirklich?

- Intralymphatische Immuntherapie: Die Zukunft hat begonnen.

— Therapieresistenter Husten: Was mache ich mit diesen Patienten?

-Molekulare Allergiediagnostik: exakt, passgenau, erfolgreich?

Neben hochkarätigen Vorträgen erwartet Sie mit dem „Hyde Park im Klostergarten" ein besonderes Diskussionsforum zu folgenden Themen: - Besonderheiten der Diagnostik von Allergien und Asthma im Kindesalter _ Entzündliche Hauterkrankungen: allergisch oder nicht?

— Allergologische Leistungen und RLV: Wege aus der Misere

Parallel zum ärztlich-wissenschaftlichen Programm findet erneut auch eine Arzthelferinnenschulung statt.

Frau Czuma und Frau Kummer Zentrum für Rhinologie und Allergologie, An den Quellen 10, 65183 Wiesbaden Tel.: (06 11) 91001530 Fax: (06 11) 8904382 E-Mail: kongressorganisation@ hno-wiesbaden.de 